\title{
SINDROME DE BURNOUT E SUAS REPERCUSSÕES NA QUALIDADE DE VIDA DE PROFESSORES E ALUNOS NO BRASIL
}

\author{
${ }^{1}$ Bruno Santana Santos, ${ }^{2}$ Cristina da Costa Oliveira, ${ }^{3}$ Pedro Fonseca de Vasconcelos, ${ }^{4}$ Mariane Costa \\ Santos de Tavares, ${ }^{5}$ Alanna Nascimento Esteves, ${ }^{6}$ Alan Vilas Boas Caetano, ${ }^{7}$ Kayky Silva Castro, \\ ${ }^{8}$ Arnaldo Neto da Cunha Bandeira, ${ }^{8}$ Ana Maria Romani, ${ }^{8}$ Jorge Vinícius Leocádio Monteiro, ${ }^{8}$ Gabriel \\ Cheles Nascimento Matos and ${ }^{9}$ Thales de Sousa Ferreira da Silva
}

\begin{abstract}
${ }^{1}$ Autor Correspondente. Cirurgião-dentista. Curso de Graduação em Medicina Faculdades Santo Agostinho, Vitória da Conquista - BA, Brasil. 2Doutora em Ciências Biológicas (Fisiologia e Farmacologia) pela Universidade Federal de Minas Gerais - UFMG. Docente do Curso de Graduação em Medicina, Faculdades Santo Agostinho, Vitória da Conquista - BA, Brasil. ${ }^{3}$ Mestre em Ciências Biológicas pela Universidade Estadual de Montes Claros - UNIMONTES. Docente do Curso de Graduação em Medicina, Faculdades Santo Agostinho, Vitória da Conquista - BA, Brasil. ${ }^{4}$ Fisioterapeuta. Especialista em

Fisioterapia Respiratória e Terapia Intensiva pelas Faculdades Integradas Pitágoras, Pós -Graduada em Deficiências Múltiplas e Sensoriais. Discente do Curso de Graduação em Medicina Faculdades Santo Agostinho, Vitória da Conquista BA, Brasil. ${ }^{5}$ Médica. Graduada em medicina pelas Faculdades Santo Agostinho, Vitória da Conquista - BA, Brasil. ${ }^{6}$ Discente do Curso de Graduação em Medicina Faculdades Santo Agostinho, Itabuna - BA, Brasil. ${ }^{7}$ Discente do Curso de Graduação em Medicina Faculdades Santo Agostinho, Vitória da Conquista - BA, Brasil. ${ }^{8}$ Discente do Curso de Graduação em Medicina Faculdades Integradas Aparício Carvalho - FIMCA. Porto Velho - RO, Brasil. ${ }^{9}$ Médico. Pós-Graduado em Gestão do Cuidado em Saúde da Família. Universidade Federal de Minas Gerais - UFMG. Belo Horizonte - MG, Brasil.
\end{abstract}

\section{ARTICLE INFO}

\section{Article History:}

Received $10^{\text {th }}$ January, 2021

Received in revised form

$16^{\text {th }}$ February, 2021

Accepted $11^{\text {th }}$ March, 2021

Published online $28^{\text {th }}$ April, 2021

\section{Key Words:}

Burnout syndrome,

Professional Exhaustion

Students,

Teachers.

*Corresponding author:

Kalile dos Anjos Souza

\begin{abstract}
Professional exhaustion promotes clinical signs and symptoms that characterize Burnout Syndrome. This situation is a consequence of an experience of chronic stress, promoting reduced quality of life, lower functionality and physical and psychological illness. The aim of this study was to identify sociodemographic, work and psychosocial factors related to the development of Burnout syndrome and impaired quality of life of students and teachers in Brazil. This is an integrative literature review, using the PICO strategy, in the databases: PubMed, Scientific Electronic Library Online - SCIELO and Latin American Literature on Health Sciences LILACS. The following Health Sciences (DeCS) Descriptors were used: "Burnout, Psychological"; "Quality of Life" and "Brazil". The results included in the results were 25 studies for analysis. The sample consisted of medical students $(40 \%)$, students from other areas of knowledge $(16 \%)$, university professors $(8 \%)$ and teachers from elementary or high schools $(36 \%)$. The prevalence of Burnout syndrome in the Brazilian academic environment ranged from 0 to $60 \%$. The specific percentages for different groups were: 12 to $44 \%$ for medical students; 0 to $10.89 \%$ among students from other areas; 30.6 to $60 \%$ for university professors; and 17.6 to $31 \%$ among elementary or high school teachers. In view of the results found, burnout syndrome has a great impact on the various actors in the student context.
\end{abstract}

Copyright (C) 2021, Bruno Santana Santos et al. This is an open access article distributed under the Creative Commons Attribution License, which permits unrestricted use, distribution, and reproduction in any medium, provided the original work is properly cited.

Citation: Bruno Santana Santos, Cristina da Costa Oliveira, Pedro Fonseca de Vasconcelos , Mariane Costa Santos de Tavares. et al. "Sindrome de burnout e suas repercussões na qualidade de vida de professores e alunos no Brasil”, International Journal of Development Research, 11, (04), 46363-46371.

\section{INTRODUCTION}

$\mathrm{N}$ os últimos anos, a saúde mental tem sido motivo de preocupação para diversas instituições de ensino e aprendizado.
(ALMEIDA et al., 2016). Segundo estimativas do Instituto Nacional de Seguridade Social do Brasil, os transtornos mentais e de comportamento são a terceira causa de incapacidade para o trabalho no país, sendo registrados 668.927 casos no período compreendido entre 2012 e 2016 (BRASIL, 2017). 
A exposição contínua ao estresse no ambiente acadêmico é um dos fatores que justificam a susceptibilidade para transtornos da saúde mental e perturbação do bem-estar biopsicossocial entre professores e alunos (ALVES; OLIVEIRA; PARO, 2019; PARO et al., 2014; PAGNIN \& QUEIROZ, 2015). Nesse cenário, a Síndrome de Burnout destaca-se como uma das desordens neuropsiquiátricas mais associadas à diminuição da qualidade de vida no meio acadêmico. (ALMEIDA et al., 2016; ALVES; OLIVEIRA; PARO, 2019; COSTA et al., 2012) Trata-se de uma doença caracterizada por uma reação anômala ao estresse emocional crônico decorrente de situações enfrentadas ao lidar rotineiramente com pessoas (BARBOSA et al., 2018). Burnout" é um termo da língua inglesa utilizado para descrever alguém ou algo que atingiu completamente os seus limites em decorrência da falta de energia (ALMEIDA et al., 2016). A síndrome é composta por três dimensões: exaustão emocional, despersonalização e realização pessoal/ satisfação profissional. (BARBOSA et al., 2018) Todos os domínios da doença afetam diretamente os componentes físicos, psicológicos, sociais e ambientais da qualidade de vida de um indivíduo (ALMEIDA et al., 2016; ALVES; OLIVEIRA; PARO, 2019; PAGNIN \& QUEIROZ, 2015).

De modo geral, a exaustão emocional é caracterizada pela sensação subjetiva de fadiga associada ao enfrentamento do trabalho. Por sua vez, a despersonalização consiste na tentativa do doente promover distanciamento afetivo e indiferença através da adoção de atitudes irônicas, frias e cínicas. Por fim, a terceira dimensão da síndrome decorre da falta de prazer com as atividades realizadas rotineiramente no ambiente de estudo/ trabalho (ALMEIDA et al., 2016).A literatura sobre o tema no Brasil ainda é escassa, dificultando analisar o real impacto da doença no contexto educacional brasileiro. Diante disto, é relevante avaliar e comparar dados obtidos por estudos nacionais para compreender o impacto da síndrome e planejar estratégias de tratamento e prevenção (BATISTA et al., 2010). O objetivo do estudo foi identificar fatores sociodemográficos, laborais e psicossociais relacionados ao desenvolvimento da síndrome de Burnout e comprometimento da qualidade de vida de discentes e docentes no Brasil.

\section{MATERIAIS E MÉTODOS}

O presente artigo recorreu a uma revisão integrativa de literatura para reunir publicações que avaliaram a Síndrome de Burnout e suas repercussões na qualidade de vida de professores e alunos no Brasil. Para guiar a revisão integrativa, a pergunta de pesquisa foi formulada utilizando a estratégia PICO, um acrônimo para "População", "Intervenção", "Comparação" e "Outcomes" (desfechos). (MENDES; SILVEIRA; GALVAO; 2019) A questão norteadora delimitada foi: "Qual o impacto da Síndrome de Burnout na qualidade de vida de professores e alunos no Brasil?". Desse modo, os elementos da estratégia PICO foram organizados da seguinte forma: P) docentes e discentes brasileiros; I) realização de uma revisão integrativa da literatura; C) não se aplica; e O) estudos que abordaram o impacto da Síndrome de Burnout na qualidade de vida da população de interesse. A pesquisa dos estudos primários foi realizada entre abril e maio de 2020, utilizando as bases de dados PubMed, SCIELO (ScientificElectronic Library Online) e LILACS (Literatura LatinoAmericana em Ciências da Saúde) empregando os seguintes Descritores em Ciências da Saúde (DeCS), na língua inglesa: "Burnout, Psychological"; "Qualityof Life" e "Brazil". O quadro 1 apresenta as estratégias de busca utilizadas e o número de referências obtidas na pesquisa inicial. Para cada estratégia de busca nas bases de dados, gerou-se um arquivo de exportação para o programa EndNote (EndNote, Thomson Reuters, versão x7) e as duplicatas foram removidas. A lista fornecida pelo EndNote foi analisada para selecionar artigos através dos títulos e resumos. Procedeu-se à seleção dos estudos em etapas, mediante aplicação de critérios de seleção. Primeiro, fez-se leitura exploratória dos títulos e, na sequência, foram lidos os resumos dos artigos escolhidos na etapa anterior. Todos os estudos selecionados foram acessados nas bases de dados on-line.
Os critérios de inclusão dos estudos foram: idioma original de publicação sendo o português, inglês ou espanhol; artigos que continham o resumo disponível on-line na íntegra indexado nas bases de dados Pubmed, SCIELO e LILACS; trabalhos que incluíram docentes e/ou discentes brasileiros em suas amostras; estudos que abordaram o Burnout e/ou suas repercussões na qualidade de vida de professores e alunos. Foram excluídos todos os trabalhos que não atenderam os critérios de seleção supracitados. Não foi delimitado critério de seleção por ano de publicação. Procedeu-se à leitura completa das referências com subsequente seleção dos artigos que atenderam os critérios de inclusão e responderam a pergunta de pesquisa.

\section{RESULTADOS}

Nesta revisão integrativa foram incluídos 25 artigos publicados entre 2006 e 2020. Mais da metade dos estudos selecionados (52\%) foram publicados nos últimos cinco anos e apenas dois foram realizados antes de 2010. Prevaleceu o idioma inglês em $80 \%$ das publicações. O processo de seleção dos estudos está representado esquematicamente na Figura 1. Identificou-se que as amostras avaliadas pelos trabalhos incluídos na revisão eram constituídas por diferentes grupos populacionais, sendo eles: alunos de medicina em $40 \%$ dos estudos $(n=10)$; estudantes de outras áreas em $16 \%(n=4)$; professores universitários em $8 \%(n=2)$; e professores de escolas do ensino fundamental ou médio em $36 \%(\mathrm{n}=9)$. O quadro 2 apresenta os estudos incluídos nesta revisão e suas principais conclusões. O Inventário de Maslach Burnout (MBI) foi a ferramenta empregada para avaliar o Burnout em todos os trabalhos que avaliaram estudantes. Nos trabalhos que incluíram professores em suas amostras, além do MBI, foram utilizadas as seguintes ferramentas: questionário de avaliação da síndrome de burnout (CESQT), Spanish burnoutinventory (SBI) e Oldenburg burnoutinventory (OLBI). O emprego de instrumentos distintos para identificar a síndrome entre docentes pode ter comprometido a qualidade dos dados obtidos pela análise comparativa de publicações nacionais. A prevalência da síndrome de Burnout no meio acadêmico brasileiro variou de 0 a $60 \%$. Os percentuais específicos para diferentes grupos foram: 12 a $44 \%$ para acadêmicos de medicina; 0 a $10,89 \%$ entre estudantes de outras áreas; 30,6 a $60 \%$ para professores universitários; e 17,6 a 31\% entre professores do ensino fundamental ou médio.

Alguns estudos transversais analisaram as dimensões física, psicológica, social e ambiental da qualidade de vida no meio acadêmico através da aplicação do questionário WHOQOL-BREF, um instrumento proposto pela Organização Mundial da Saúde (OMS) para quantificar o bem estar biopsicossocial de indivíduos. O quadro 3 apresenta os escores obtidos na aplicação do referido questionário entre docentes e discentes do Brasil. Ressalta-se que, as variações observadas nas diferentes regiões do Brasil, tanto para prevalência de Burnout, quanto para escores no questionário WHOQOL-BREF, podem estar diretamente relacionadas com aspectos culturais, socioeconômicos e ocupacionais do ambiente acadêmico em que professores e alunos estão inseridos. Os dados obtidos pelos trabalhos apresentados no quadro 3 revelaram que o Burnout e suas dimensões são importantes preditores negativos de qualidade de vida no meio acadêmico. As três dimensões da síndrome foram correlacionadas negativamente com o bem estar biopsicossocial de professores e alunos (ALMEIDA et al., 2016 ; COSTA et al., 2012; PAGNIN \& QUEIROZ, 2015; TABELEÃO; TOMASI; NEVES, 2011). O sexo feminino foi a variável demográfica mais associada com diminuição da qualidade de vida entre docentes e discentes do Brasil. Esse achado parece ser resultado das maiores prevalências de Burnout e exaustão emocional em mulheres do que em homens. (ALVES; OLIVEIRA; PARO, 2019; BATISTA et al., 2010; BARBOSA et al., 2018; Costa \& Silva, 2012; PARO et al., 2014; TABELEÃO; TOMASI; NEVES, 2011). Os escores de qualidade de vida foram diretamente relacionados com a sensação de eficácia acadêmica/ realização profissional (ALVES; OLIVEIRA; PARO, 2019; PAGNIN \& QUEIROZ, 2015; PARO et al., 2014) e inversamente associados com a exaustão física decorrente da sobrecarga ocupacional. 
Quadro 1. Estratégias de busca nas bases de dados PubMed, SCIELO e LILACS

\begin{tabular}{llll}
\hline & \multicolumn{3}{c}{ Número de artigosencontrados } \\
\cline { 2 - 4 } Estratégia de busca & Pubmed & SCIELO & LILACS \\
"Burnout, Psychological" \& "Quality of Life” & 987 & 25 & 9 \\
"Burnout, Psychological" \& "Brazil” & 182 & 36 & 15 \\
TOTAL: & 1.169 & 61 & 24 \\
\hline
\end{tabular}

Quadro 2. Estudos selecionados para revisão integrativa e suas principais conclusões

\begin{tabular}{|c|c|c|c|c|c|}
\hline Estudo & Local & Amostra & $\begin{array}{l}\text { Ferramen } \\
\text { ta }\end{array}$ & Tipo de estudo & PrincipaisConclusões \\
\hline Almeida et al. (2016) & Ceará & $\begin{array}{l}517 \text { alunos de medicina do } 1^{\circ} \\
\text { ao } 8^{\circ} \text { período }\end{array}$ & MBI & Transversal & $\begin{array}{l}14,9 \% \text { de prevalência para síndrome de } \\
\text { Burnout na amostra avaliada. }\end{array}$ \\
\hline $\begin{array}{l}\text { Alves, Oliveira e Paro } \\
(2019)\end{array}$ & $\begin{array}{l}\text { Minas } \\
\text { Gerais }\end{array}$ & 366 professoresuniversitários & OLBI & Transversal & $\begin{array}{l}\text { O Burnout associou-se negativamente com a } \\
\text { qualidade de vida dos docentes. }\end{array}$ \\
\hline Araújo et al. (2020) & $\begin{array}{l}\text { Rio Grande } \\
\text { do Norte }\end{array}$ & 100 professoresuniversitários & MBI & Transversal & $\begin{array}{l}60 \% \text { de prevalência para síndrome de Burnout } \\
\text { na amostra avaliada. . }\end{array}$ \\
\hline Barbosa et al. (2018) & Goiás & $\begin{array}{l}399 \text { alunos de medicina dos } \\
\text { quatro primeiros anos do curso }\end{array}$ & MBI & Transversal & $\begin{array}{l}\text { O quinto período do curso e o sexo feminino } \\
\text { foram mais afetados pelo Burnout. }\end{array}$ \\
\hline Batista et al. (2010) & Paraíba & 265 professores de escolas & MBI & Transversal & $\begin{array}{l}\text { Intervenções que atuem sobre fatores laborais } \\
\text { e psicossociais podem prevenir o Burnout. }\end{array}$ \\
\hline Boniet al. (2018) & São Paulo & $\begin{array}{l}265 \text { alunos de medicina dos } \\
\text { quatro primeiros anos do curso }\end{array}$ & MBI & Transversal & $\begin{array}{l}\text { Otimismo, autopercepção de saúde, motivação } \\
\text { e rotina de estudos estão associados aos níveis } \\
\text { de Burnout. }\end{array}$ \\
\hline Calcideset al. (2019) & Sergipe & $\begin{array}{l}184 \text { alunos de medicina dos } \\
\text { dois últimos anos do curso }\end{array}$ & MBI & Transversal & $\begin{array}{l}35,9 \% \text { de prevalência para síndrome de } \\
\text { Burnout na amostra avaliada (utilizando o } \\
\text { critério bidimensional). }\end{array}$ \\
\hline $\begin{array}{l}\text { Campos } \\
\text { \&Maroco (2012) }\end{array}$ & $\begin{array}{l}\text { Brasil e } \\
\text { Portugal }\end{array}$ & $\begin{array}{l}1.514 \text { estudantes universitários } \\
(958 \text { brasileiros e } 556 \\
\text { portugueses) }\end{array}$ & MBI & Transversal & $\begin{array}{l}\text { O questionário Maslach Burnout Inventory } \\
(\mathrm{MBI}) \text { é uma ferramenta confiável para } \\
\text { rastrear a síndrome de Burnout }\end{array}$ \\
\hline $\begin{array}{l}\text { Carlotto \& Palazzo } \\
(2006)\end{array}$ & $\begin{array}{l}\text { Rio Grande } \\
\text { do Sul }\end{array}$ & 190 professores de escolas & MBI & Transversal & $\begin{array}{l}\text { A falta de participação em decisões } \\
\text { institucionais esta associada com o Burnout. }\end{array}$ \\
\hline $\begin{array}{l}\text { Carlotto \& Câmara } \\
(2017)\end{array}$ & $\begin{array}{l}\text { Rio Grande } \\
\text { do Sul }\end{array}$ & 679 professores de escolas & SBI & Transversal & $\begin{array}{l}\text { O conflito de papéis e a intenção de deixar a } \\
\text { profissão são fortemente correlacionados com } \\
\text { o Burnout. }\end{array}$ \\
\hline $\begin{array}{ll}\text { Costa } & \& \\
\text { Silva }(2012) & \end{array}$ & Paraná & 100 professores de escolas & MBI & Transversal & $\begin{array}{l}\text { Os sintomas da síndrome são muito } \\
\text { prevalentes tanto em professores mais jovens } \\
\text { quanto naqueles com maior experiência } \\
\text { profissional. }\end{array}$ \\
\hline Costa et al. (2012) & Sergipe & $\begin{array}{l}369 \text { alunos de medicina (todos } \\
\text { os períodos) }\end{array}$ & MBI & Transversal & $\begin{array}{l}\text { Fatores diretamente relacionados ao processo } \\
\text { de educação médica estão associados ao } \\
\text { Burnout. }\end{array}$ \\
\hline $\begin{array}{l}\text { Giannini; Latorre e } \\
\text { Ferreira (2013) }\end{array}$ & São Paulo. & $\begin{array}{l}354 \text { professores de escolas } \\
\text { (todos do sexo feminino) }\end{array}$ & - & Caso-controle & $\begin{array}{l}\text { Existe relação entre estresse ocupacional, } \\
\text { distúrbios da voz e perda da capacidade para o } \\
\text { trabalho. }\end{array}$ \\
\hline $\begin{array}{l}\text { Gil-Monte; Carlotto e } \\
\text { Câmara (2011) }\end{array}$ & $\begin{array}{l}\text { Rio Grande } \\
\text { do Sul }\end{array}$ & 714 professores de escolas & SBI & Transversal & $\begin{array}{l}17,6 \% \text { de prevalência para síndrome de } \\
\text { Burnout na amostra avaliada. }\end{array}$ \\
\hline Lopes \& Nihei (2020) & Paraná & $\begin{array}{l}284 \text { alunos de enfermagem do } \\
\text { terceiro, quarto e quinto ano }\end{array}$ & MBI & Transversal & $\begin{array}{l}\text { Ausência de atividade física, carga } \\
\text { ocupacional semanal e baixa preocupação } \\
\text { empática foram preditores da síndrome de } \\
\text { burnout. }\end{array}$ \\
\hline Motaet al. (2019) & Sergipe & 208 professores de escolas & CESQT & Transversal & $\begin{array}{l}\text { O Burnout é positivamente associado a } \\
\text { distúrbios da voz entre professores }\end{array}$ \\
\hline $\begin{array}{lll}\text { Neves } & \& & \text { Ribeiro } \\
(2016) & & \\
\end{array}$ & $\begin{array}{l}\text { Santa } \\
\text { Catarina }\end{array}$ & $\begin{array}{l}105 \text { alunos de odontologia do } \\
1^{\circ}, 5^{\circ} \text { e } 10^{\circ} \text { períodos }\end{array}$ & MBI & Transversal & $10,89 \%$ de prevalência do Burnout \\
\hline Pacheco et al. (2017) & $\begin{array}{l}\text { Brasil } \\
\text { (váriosestad } \\
\text { os) }\end{array}$ & $\begin{array}{l}59 \text { estudos envolvendo um total } \\
\text { de } 18.015 \text { alunos de medicina }\end{array}$ & MBI & $\begin{array}{l}\text { Revisãosistemá } \\
\text { tica }\end{array}$ & $\begin{array}{l}\text { A prevalência média da síndrome de Burnout } \\
\text { entre alunos de medicina no Brasil é de } 13,1 \%\end{array}$ \\
\hline $\begin{array}{l}\text { Pagnin\& } \quad \text { Queiroz } \\
(2015)\end{array}$ & $\begin{array}{l}\text { Rio de } \\
\text { Janeiro }\end{array}$ & $\begin{array}{l}193 \text { alunos de medicina do } \\
\text { segundo ano }\end{array}$ & MBI & Transversal & $\begin{array}{l}\text { As dimensões do Burnout correlacionam-se } \\
\text { com comprometimento da qualidade de vida } \\
\text { de discentes. }\end{array}$ \\
\hline Paro et al. (2014) & $\begin{array}{l}\text { Brasil } \\
\text { (váriosestad } \\
\text { os) }\end{array}$ & $\begin{array}{l}1.650 \quad \text { alunos } \\
\text { escolasmédicas }\end{array}$ & MBI & $\begin{array}{l}\text { Transversal e } \\
\text { multicêntrico }\end{array}$ & $\begin{array}{l}\text { Existem associações inversas significativas } \\
\text { entre empatia e síndrome de Burnout. }\end{array}$ \\
\hline $\begin{array}{l}\text { Ramos-Dias et al. } \\
(2010)\end{array}$ & São Paulo & $\begin{array}{l}100 \text { alunos de medicina do } \\
\text { primeiro e último ano do curso }\end{array}$ & - & Transversal & $\begin{array}{l}\text { Alunos do primeiro ano apresentam menor } \\
\text { qualidade de vida do que estudantes do sexto } \\
\text { ano. }\end{array}$ \\
\hline Reis et al. (2006) & Bahia & 808 professores de escolas & - & Transversal & $\begin{array}{l}\text { O grau de exigência no trabalho associa-se } \\
\text { com as prevalências de cansaço mental e de } \\
\text { nervosismo entre professores. }\end{array}$ \\
\hline $\begin{array}{l}\text { Solis \&Lotufo-Neto } \\
(2019)\end{array}$ & $\begin{array}{l}\text { Brasil } \\
\text { (váriosestad } \\
\text { os) }\end{array}$ & $\begin{array}{l}31 \text { estudos envolvendo um total } \\
\text { de } 3.402 \text { alunos de medicina } \\
\text { (todos os períodos) }\end{array}$ & MBI & $\begin{array}{l}\text { Revisãosistemá } \\
\text { tica }\end{array}$ & $\begin{array}{l}\text { O sexo feminino é um importante preditor } \\
\text { negativo de qualidade de vida nos domínios da } \\
\text { saúde física e psicológica. }\end{array}$ \\
\hline $\begin{array}{l}\text { Tabeleão; Tomasi; } \\
\text { Neves, (2011) }\end{array}$ & $\begin{array}{l}\text { Rio Grande } \\
\text { do Sul }\end{array}$ & 601 professores de escolas & MBI & Transversal & $\begin{array}{l}\text { Sexo masculino e a maior renda familiar foram } \\
\text { preditores de melhor qualidade de vida. }\end{array}$ \\
\hline $\begin{array}{l}\text { Tomaschewski- } \\
\text { barlemiet al. }(2014)\end{array}$ & $\begin{array}{l}\text { Rio Grande } \\
\text { do Sul }\end{array}$ & $\begin{array}{l}168 \text { alunos de enfermagem do } \\
1^{\circ} \text { ao } 9^{\circ} \text { período }\end{array}$ & MBI & Transversal & $\begin{array}{l}\text { Nenhum estudante de enfermagem apresentou } \\
\text { a síndrome de Burnout. }\end{array}$ \\
\hline
\end{tabular}


Quadro 3. Escores obtidos por estudos transversais que aplicaram o questionário WHOQOL-BREF no Brasil e preditores de qualidade de vida identificados

\begin{tabular}{|c|c|c|c|c|c|}
\hline Autor & $\begin{array}{l}\text { Amostra } \\
(\mathrm{n})\end{array}$ & Local do estudo & WHOQOL-BREF (pontuações) & Preditorespositivos & $\begin{array}{l}\text { Preditores } \\
\text { negativos }\end{array}$ \\
\hline $\begin{array}{l}\text { Paro et al. } \\
(2014)\end{array}$ & $\begin{array}{l}1.650 \\
\text { alunos }\end{array}$ & $\begin{array}{l}22 \text { escolasmédicas } \\
\text { (diferentesestados } \\
\text { ) }\end{array}$ & $\begin{array}{l}\text { Físico: } 65,2 \\
\text { Psicológico: } 61,7 \\
\text { Relações sociais: } 63,6 \\
\text { Ambiental: } 63,8\end{array}$ & $\begin{array}{l}\text { Realização pessoal; } \\
\text { preocupaçãoempática. }\end{array}$ & $\begin{array}{l}\text { Exaustão emocional; } \\
\text { despersonalização; } \\
\text { angústia pessoal; sexo } \\
\text { feminino. }\end{array}$ \\
\hline $\begin{array}{l}\text { Ramos-Dias et } \\
\text { al. }(2010)\end{array}$ & $\begin{array}{l}100 \\
\text { alunos }\end{array}$ & $\begin{array}{lr}\text { Universidade } & \\
\text { Católica } & \text { de } \\
\text { Sorocaba } & \text { (São } \\
\text { Paulo) } & \\
\end{array}$ & $\begin{array}{l}\text { Físico*: } 77,9 \text { e } 76,8 \\
\text { Psicológico*: } 71,1 \text { e } 72,6 \\
\text { Relações sociais*: 70,2 e 77,8 } \\
\text { Ambiental*: } 67,7 \text { e } 70,1\end{array}$ & & $\begin{array}{l}\text { Primeiro ano da graduação } \\
\text { em medicina }\end{array}$ \\
\hline $\begin{array}{l}\text { Pagnin\& } \\
\text { Queiroz (2015) }\end{array}$ & $\begin{array}{l}193 \\
\text { alunos }\end{array}$ & $\begin{array}{l}\text { Universidade } \\
\text { Federal } \\
\text { Fluminense (Rio } \\
\text { de Janeiro) }\end{array}$ & $\begin{array}{l}\text { Físico: } 59,55 \\
\text { Psicológico: } 62,36 \\
\text { Relações sociais: } 66,97 \\
\text { Ambiental: } 61,97 \\
\end{array}$ & $\begin{array}{l}\text { Senso de eficácia; } \\
\text { realização acadêmica. }\end{array}$ & $\begin{array}{l}\text { Burnout; Problemas do } \\
\text { sono; Cinismo; Sintomas } \\
\text { depressivos. }\end{array}$ \\
\hline $\begin{array}{l}\text { Tabeleão, } \\
\text { Tomasi e Neves } \\
(2011)\end{array}$ & $\begin{array}{l}601 \\
\text { professor } \\
\text { es }\end{array}$ & $\begin{array}{l}105 \text { escolas (Rio } \\
\text { Grande do Sul) }\end{array}$ & $\begin{array}{l}\text { Físico: } 69,2 \\
\text { Psicológico: } 70,6 \\
\text { Relações sociais: } 72,5 \\
\text { Ambiental: } 60,7\end{array}$ & $\begin{array}{l}\text { Sexo masculino; maior } \\
\text { renda familiar. }\end{array}$ & $\begin{array}{l}\text { Burnout; atuar em escolas } \\
\text { públicas estaduais. }\end{array}$ \\
\hline $\begin{array}{l}\text { Alves, Oliveira } \\
\text { e Paro (2019) }\end{array}$ & $\begin{array}{l}366 \\
\text { professor } \\
\text { es }\end{array}$ & $\begin{array}{l}\text { Universidade } \\
\text { Federal de } \\
\text { Uberlândia, } \\
\text { (Minas Gerais) }\end{array}$ & $\begin{array}{l}\text { Físico: } 71,13 \\
\text { Psicológico: } 71,63 \\
\text { Relações sociais: } 67,4 \\
\text { Ambiental: } 65,1\end{array}$ & & $\begin{array}{l}\text { Burnout; sexo feminino; } \\
\text { maior necessidade de } \\
\text { tempo para relaxar após o } \\
\text { trabalho. }\end{array}$ \\
\hline
\end{tabular}

*Pontuações obtidas para o $1^{\circ}$ e $6^{\circ}$ anos, respectivamente.

(ALMEIDA et al., 2016; ALVES; OLIVEIRA; PARO, 2019; BARBOSA et al., 2018; BATISTA et al., 2010; PACHECO et al., 2017) A forma com que a instituição de ensino lida com professores e alunos influenciou no bem estar destes sujeitos. (ALVES; OLIVEIRA; PARO, 2019; BATISTA et al., 2010; PAGNIN \& QUEIROZ, 2015). Estudos nacionais revelaram que o primeiro ano da graduação em medicina tanto é um preditor negativo da qualidade de vida no domínio de relações sociais (RAMOS-DIAS et al., 2010) quanto se associa com níveis aumentados de Burnout (BONI et al., 2018). Outros trabalhos encontraram que o pico de incidência da doença ocorre no último ano do curso médico (ALMEIDA et al., 2016; COSTA et al., 2012). O comprometimento do bem-estar psicológico no meio acadêmico foi associado com frequência aos estressores ocupacionais envolvidos com o desenvolvimento da síndrome de Burnout. (ALMEIDA et al., 2016 ; ALVES; OLIVEIRA; PARO, 2019; CARLOTTO \& PALAZZO, 2006; COSTA et al., 2012; GIL-MONTE; CARLOTTO; CÂMARA, 2011; PACHECO et al., 2017).

Sintomas depressivos (PAGNIN \& QUEIROZ, 2015), escores de sofrimento pessoal e falta de preocupação empática (PARO et al., 2014 foram preditores negativos da qualidade de vida no ambiente universitário. Essas três variáveis psicológicas também estiveram correlacionadas com o Burnout (BARBOSA et al., 2018; COSTA et al., 2012; LOPES \& NIHEI, 2020; PACHECO et al., 2017; PARO et al., 2014). O relato de doenças crônicas (SOLIS \& LOTUFO-NETO, 2019), distúrbios do sono (PAGNIN \& QUEIROZ, 2015; SOLIS \& LOTUFO-NETO, 2019) e a necessidade de mais tempo para relaxar após o trabalho (ALVES; OLIVEIRA; PARO, 2019) foram outros preditores negativos da qualidade de vida no ambiente acadêmico. (SOLIS \& LOTUFO-NETO, 2019). A exaustão emocional e a resposta anômala ao estresse crônico que ocorrem na síndrome de Burnout correlacionam-se positivamente com o comprometimento da saúde física de professores e alunos (ALVES; OLIVEIRA; PARO, 2019; BARBOSA et al., 2018; Costa \& Silva, 2012; PAGNIN \& QUEIROZ, 2015) Autopercepção de saúde (BONI et al., 2018), história de afastamento do trabalho por motivo de saúde (BATISTA et al., 2010) e relato de doenças crônicas (ARAÚJO et al., 2020) foram variáveis positivamente associada com a doença. Fatores associados com a dimensão "relações sociais" da qualidade de vida também influenciaram no desenvolvimento do Burnout. Assim, baixa frequência de encontros familiares, tempo de lazer e influência da profissão na vida pessoal foram variáveis de susceptibilidade para síndrome (BONI et al., 2018; BATISTA et al., 2010; CALCIDES et al., 2019).

\section{DISCUSSÃO}

Prevalência e instrumentos para avaliação do Burnout entre alunos: Ao analisar a literatura nacional sobre o Burnout entre estudantes, percebe-se que a doença tem sido pesquisada com maior frequência entre acadêmicos de medicina do que em outros alunos de graduação. Isso parece indicar que estudantes que ingressam no curso médico são mais expostos a fatores estressores que podem desencadear o Burnout. Dados obtidos por estudos brasileiros demonstram que, quando comparados com pares da mesma idade na população geral, os alunos do curso médico apresentam a síndrome de Burnout com maior frequência. (BARBOSA et al., 2018; COSTA et al., 2012; PACHECO et al., 2017). A maioria dos estudos nacionais utilizou uma versão adaptada do Maslach Burnout Inventory (MBI) para identificar o Burnout. Campos \&Maroco (2012) demonstraram que a ferramenta em questão é confiável para rastrear a doença entre estudantes universitários do Brasil. (CAMPOS \& MAROCO, 2012). $\mathrm{O}$ MBI é um questionário de auto-relato composto por 15 perguntas que avaliam os três domínios da síndrome (ARAÚJO et al., 2020). Cada item do questionário é pontuado de acordo com sua frequência (0 a 6 pontos) utilizando a escala Likert. Assim, são atribuídas as seguintes pontuações para cada uma das dimensões do Burnout: exaustão emocional ( 0 a 54 pontos), despersonalização (faixa de 0 a 30 pontos) e realização pessoal ( 0 a 48 pontos). (ARAÚJO et al., 2020; COSTA et al., 2012; CALCIDES et al., 2019).

O escore é positivo quando são atingidos mais de 14 pontos na exaustão emocional, mais de 6 pontos na despersonalização e menos de 23 pontos na realização pessoal. $O$ diagnóstico pode ser considerado quando as três dimensões atingem as pontuações alvo (critério tridimensional) ou quando apenas as dimensões exaustão e cinismo são positivas (critério bidimensional). (CALCIDES et al., 2019). Foram identificadas taxas de prevalência semelhantes para o Burnout entre estudantes do curso médico nos estados do Ceará $(14,9 \%)$ (ALMEIDA et al., 2016) e de Goiás (12,0\%) (BARBOSA et al., 2018). Uma metanálise com 59 estudos que analisou um total de 18.015 acadêmicos de medicina no Brasil identificou prevalência de $13,1 \%$ para o Burnout (PACHECO et al., 2017). Os maiores índices diagnósticos da doença no território nacional foram encontrados entre acadêmicos de medicina de uma faculdade particular de São Paulo que utilizava metodologias ativas de ensino. A prevalência da síndrome variou conforme o critério empregado para definir o Burnout, sendo de $44,9 \%$ no método bidimensional e $26,4 \%$ no critério tridimensional (BONI et al., 2018). 
Calcides et al. (2019) também avaliaram de forma distinta os percentuais encontrados por diferentes interpretações dos critérios diagnósticos da síndrome numa escola médica do nordeste. Os percentuais identificados foram $35,9 \%$ nos critérios bidimensionais e $10,3 \%$ nos tridimensionais. (CALCIDES et al., 2019). Embora a literatura nacional abordando o Burnout entre estudantes de outros cursos de graduação que não o de medicina seja escassa, a análise dos dados obtidos até o momento demonstra que a prevalência da doença é significativamente menor entre acadêmicos de enfermagem. (LOPES \& NIHEI, 2020; NEVES \& RIBEIRO, 2016; TOMASCHEWSKI-BARLEMI et al., 2014). No estudo de Tomaschewski-Barlemi et al. (2014), realizado no Rio Grande do Sul, nenhum dos 168 estudante de enfermagem avaliados apresentaram o diagnóstico da doença. Lopes \&Nihei (2020) também realizaram um estudo transversal com acadêmicos de enfermagem e identificaram $6,0 \%$ de prevalência do Burnout entre alunos de uma instituição localizada no Paraná (LOPES \& NIHEI, 2020). Ao investigar a presença da síndrome entre acadêmicos de odontologia em Santa Catarina, Neves \& Ribeiro (2016) identificaram taxas de prevalência superiores àquelas encontradas entre estudantes de enfermagem, porém menores do que às encontradas no curso médico. A síndrome foi diagnosticada em 10,89\% dos alunos avaliados (NEVES \& RIBEIRO, 2016). Conclui-se, portanto, que o Burnout apresenta alta prevalência entre acadêmicos de medicina de todo o território nacional. Assim, percebe-se que é de extrema relevância que as escolas médicas brasileiras desenvolvam estratégias de monitoramento e gestão de fatores acadêmicos e institucionais associados ao desenvolvimento da síndrome no transcorrer do curso médico.

Prevalência e instrumentos para avaliação do Burnout entre professores: A categoria docente é uma das mais investigadas em termos de Burnout pela literatura nacional. O número de publicações abordando a doença entre professores brasileiros tem aumentado consideravelmente nos últimos anos, o que demonstra uma preocupação crescente com a saúde mental destes profissionais. Os autores que investigaram a prevalência do Burnout entre docentes do Brasil aplicaram ferramentas distintas para identificar a presença da doença, o que pode comprometer a acurácia dos dados advindos da análise comparativa de estudos nacionais. Os questionários empregados para identificar a síndrome foram: Maslach Burnout Inventory-EducatorsSurvey (MBI-ED), Oldenburg Burnout Inventory (OLBI) e Spanish Burnout Inventory (SBI) (ARAÚJO et al., 2020; ALVES; OLIVEIRA; PARO, 2019; CARLOTTO \& CÂMARA, 2017). O MBI-ED é uma versão do inventário de Maslach adaptada para professores que define o diagnóstico de Burnout quando se obtêm 56 ou mais pontos na aplicação de um questionário composto por 22 perguntas (pontuadas de 1 a 5 ) que avaliam as três dimensões da síndrome (ARAÚJO et al., 2020). Por sua vez, o OLBI identifica a presença da síndrome através de um questionário que inclui itens de exaustão ( 3 perguntas) e desengajamento (6 perguntas). Cada uma das respostas é pontuada de 0 a 4 pontos, sendo que, escores médios elevados nas dimensões exaustão (maior ou igual a 2,25) e desengajamento (maior ou igual a 2,1) indicam a presença do Burnout (ALVES; OLIVEIRA; PARO, 2019). O SBI é uma ferramenta que identifica a doença mediante aplicação de um questionário composto por 20 perguntas que avaliam as seguintes variáveis: entusiasmo pelo trabalho, exaustão psicológica, indolência e culpa. As respostas são pontuadas numa escala de frequência ( 0 a 4 pontos) e indivíduos com escores maiores ou iguais ao percentil 90 são identificados como sendo portadores do Burnout (CARLOTTO \& CÂMARA, 2017). O emprego de diferentes ferramentas para identificar a síndrome pode ter influenciado nas variações de prevalência encontradas por estudos nacionais. A análise da literatura brasileira demonstra que, majoritariamente, os professores universitários apresentaram maiores taxas de Burnout do que profissionais que atuam em escolas de ensino fundamental e médio. Alves, Oliveira e Paro (2019) demonstraram que o Burnout estava presente e afetava a qualidade de vida de $36,6 \%$ dos professores de uma universidade pública em Minas Gerais (ALVES; OLIVEIRA; PARO, 2019). Outro trabalho que também avaliou docentes universitários identificou o diagnóstico da síndrome em cerca de $60 \%$ dos profissionais que atuavam no nordeste do
Brasil. (ARAÚJO et al., 2020). Gil-Monte; Carlotto e Câmara (2011) demonstraram que a doença é menos comum entre professores que atuam no ensino fundamental e/ou médio do Rio Grande do Sul, sendo identificada prevalência de 17,6\%. (GIL-MONTE; CARLOTTO; CÂMARA, 2011). Tabeleão, Tomasi e Neves (2011), também avaliando professores que atuam em escolas no Sul do Brasil, encontraram $31 \%$ de prevalência para o burnout (TABELEÃO; TOMASI; NEVES, 2011). Portanto, a análise dos resultados obtidos por estudos epidemiológicos permitem inferir que, assim como acadêmicos de medicina, a categoria docente encontra-se susceptível à síndrome de Burnout. Diante disso, é possível concluir que o ambiente acadêmico nacional alberga diversos fatores estressores associados ao comprometimento da saúde mental de professores e alunos no Brasil.

Burnout e qualidade de vida entre docentes e discentes do Brasil: Alguns estudos nacionais quantificaram a qualidade de vida de professores e alunos através da aplicação de um questionário específico para este fim, o WHOQOL-BREF. Esse último é um instrumento proposto pela Organização Mundial da Saúde (OMS) para avaliar a qualidade de vida de diferentes grupos populacionais. O WHOQOL-BREF é um questionário composto por 26 perguntas que mensuram a percepção de um indivíduo sobre sua saúde e qualidade de vida nos domínios físico, psicológico, sociais e ambiental. As respostas obtidas para cada domínio são convertidas para uma escala de 0 a 100, sendo que, quanto maior o escore final obtido, melhor é a percepção de qualidade de vida do sujeito avaliado. (PAGNIN \& QUEIROZ, 2015). Dentre todos os domínios avaliados pelo questionário WHOQOL-BREF (físico, psicológico, relações sociais e ambiente), as menores pontuações entre professores foram observados no domínio ambiente. Em contrapartida, considerando alunos de medicina, os menores escores encontrados pelo estudo com amostra mais representativa $(n=1650)$ ocorreram no domínio psicológico. Dois estudos que mensuraram a qualidade de vida entre professores brasileiros empregando o WHOQOL-BREF encontraram pontuações superiores àquelas encontradas entre alunos de medicina por Pagnin\& Queiroz (2015) e Paro et al. (2014) nos domínios físico, psicológico e relações sociais.

O único trabalho nacional com estudantes de medicina que identificou pontuações superiores àquelas encontradas entre professores aplicando o questionário WHOQOL-BREF foi realizado por RamosDias et al. (2010), no estado de são Paulo. Ressalta-se que, o estudo em questão incluiu apenas 100 alunos de uma escola médica particular em sua amostra, o que pode ter influenciado nos resultados obtidos. Especificamente no domínio ambiente, foram identificadas pontuações semelhantes no WHOQOL-BREF entre docentes e discentes do Brasil. Ao analisar uma amostra considerável de professores $(\mathrm{n}=601)$, Tabeleão, Tomasi e Neves (2011) encontraram escores no domínio ambiente mais baixos do aqueles obtidos por três trabalhos que avaliaram alunos de medicina. As três dimensões da síndrome de Burnout correlacionam-se fortemente com o comprometimento da qualidade de vida de professores e alunos (ALMEIDA et al., 2016 ; COSTA et al., 2012; PAGNIN \& QUEIROZ, 2015). Tabeleão, Tomasi e Neves (2011) demonstraram que todas as dimensões da síndrome, sem exceções, estavam associadas com menores pontuações nos escores de qualidade de vida de professores que atuam em escolas do Sul do Brasil. (TABELEÃO; TOMASI; NEVES, 2011) Por sua vez, Pagnin\& Queiroz (2015) constataram que as três dimensões do Burnout também estão correlacionadas com menores pontuações no questionário WHOQOLBREF entre alunos do curso médico. (PAGNIN \& QUEIROZ, 2015). Desse modo, percebe-se que a síndrome de Burnout e suas dimensões são importantes preditores negativos da qualidade de vida no ambiente acadêmico, o que foi demonstrando por diversos trabalhos que aplicaram o questionário WHOQOL-BREF entre professores e alunos. Assim, emerge a necessidade de desenvolver estratégias para atenuar os impactos da referida doença no bem estar biopsicossocial de docentes e discentes do Brasil.

Fatores sociodemográficos: Estudos transversais demonstraram que o sexo feminino é um preditor negativo de qualidade de vida entre 
discentes e docentes no Brasil (ALVES; OLIVEIRA; PARO, 2019; PARO et al., 2014; Tabeleão; Tomasi; Neves, 2011). Essa associação foi verificada tanto em professores que atuam no ensino fundamental e médio (TABELEÃO; TOMASI; NEVES, 2011 quanto entre membros de um corpo docente universitário (ALVES; OLIVEIRA; PARO, 2019). Entre professores universitários, o sexo feminino esteve relacionado com menor percepção de todos os domínios avaliados pelo WHOQOL (ALVES; OLIVEIRA; PARO, 2019). Em contrapartida, para profissionais que atuam em escolas do ensino fundamental e médio, a mesma associação só foi constatada nos domínios físico e psicológico (TABELEÃO; TOMASI; NEVES, 2011). Houve uma forte correlação entre os níveis de Burnout e a qualidade de vida de docentes, independente de sua área de atuação (ALVES; OLIVEIRA; PARO, 2019; Tabeleão; Tomasi; Neves, 2011) Ao avaliar escolas médicas de diferentes estados do Brasil, Paro et al. (2014) demonstram que o sexo feminino é negativamente associado com os escores de qualidade de vida física e psicológica dos sujeitos avaliados (PARO et al., 2014) A relação entre sexo feminino e comprometimento da saúde física e psicológica de acadêmicos de medicina também foi observada na revisão sistemática realizada por Solis\& Lotufo-Neto (2019).

Sugere-se que, enquanto os homens dão menos importância aos seus sintomas físicos e psicológicos, as mulheres respondem mais criticamente aos instrumentos de avaliação e, por isso, tendem a apresentar menores escores de bem-estar biopsicossocial (ALVES; OLIVEIRA; PARO, 2019). Outra explicação proposta para explicar as referidas diferenças de gênero é a de que o domínio dos homens nos cargos universitários de maior poder contribua para o aumento da exaustão emocional e diminuição da percepção da qualidade de vida entre professoras (ALVES; OLIVEIRA; PARO, 2019). Batista et al. (2010) demonstraram associação entre titulação profissional e síndrome de Burnout entre docentes, o que corrobora com a hipótese supracitada (BATISTA et al., 2010). Costa \& Silva (2012) observaram que, quando comparadas com indivíduos do sexo masculino, as mulheres que atuam na docência são mais susceptíveis à exaustão emocional (Costa \& Silva, 2012). Essa última, por sua vez, foi considerada um preditor negativo da qualidade de vida de professores e alunos de medicina (ALVES; OLIVEIRA; PARO, 2019; Paro et al., 2014). A exaustão emocional também foi fortemente correlacionada com a gênese da síndrome de Burnout entre professores (ALVES; OLIVEIRA; PARO, 2019) e com o sofrimento pessoal de alunos (PARO et al., 2014). Portanto, a susceptibilidade do sexo feminino à exaustão emocional parece explicar as menores pontuações em escores que mensuram o bem estar biopsicossocial de docentes e discentes. Diversos trabalhos nacionais demonstraram que, quando comparadas com indivíduos do sexo masculino, as mulheres apresentam maior prevalência da síndrome de Burnout (ALVES; OLIVEIRA; PARO, 2019, BATISTA et al., 2010; BARBOSA et al., 2018).

Esses resultados reforçam a hipótese de que professoras e alunas realmente apresentam menor percepção do seu bem estar biopsicossocial, pois, existem fortes evidências da associação inversa do Burnout com a qualidade de vida de professores e alunos no Brasil. O status socioeconômico é outro fator que pode influenciar na qualidade de vida de professores e alunos. Tabeleão, Tomasi e Neves (2011) identificaram que, quanto maior a renda familiar, mais alta é a pontuação obtida por docentes em todos os domínios avaliados no questionário WHOQOL (TABELEÃO; TOMASI; NEVES, 2011). Nesse cenário, sugere-se que as pressões diárias advindas do baixo status socioeconômico possam afetar a saúde mental de professores, o que explicaria a diminuição do bem estar biopsicossocial observada nestes profissionais (SOLIS; LOTUFO-NETO, 2019). A distância entre instituição de ensino e cidade natal do aluno foi outra variável demográfica identificada como um preditor negativo de qualidade de vida (SOLIS; LOTUFO-NETO, 2019). A migração de jovens para longe de suas famílias também se associa com aumento do risco de Burnout entre estudantes de medicina (BARBOSA et al. 2018). Idade avançada, ausência de companheiro fixo e não possuir filhos foram fatores demográficos associados a risco aumentado para o Burnout entre professores (ALVES; OLIVEIRA; PARO, 2019, BATISTA et al. 2010). Embora essas variáveis não tenham sido diretamente identificadas como preditores negativos de qualidade de vida, deve-se considerar a possibilidade de que essa associação ocorra de forma indireta, como resultado das repercussões biopsicossociais do Burnout.

Fatores acadêmicos e ocupacionais: Diversos estudos demonstraram que a exaustão física resultante da sobrecarga ocupacional é um importante fator de risco para a diminuição da percepção geral da qualidade de vida de professores e alunos (ALMEIDA et al., 2016; ALVES; OLIVEIRA; PARO, 2019; BARBOSA et al., 2018; BATISTA et al., 2010; PACHECO et al., 2017) Ressalta-se que, o efeito dos estressores ocupacionais pode ser potencializado por traços de personalidade que incluem perfeccionismo, obstinação e comportamentos obsessivos. (ALMEIDA et al., 2016; BARBOSA et al., 2018). Carga excessiva de estudo/trabalho, estresse ocupacional e vivência em ambientes competitivos foram variáveis laborais associadas ao Burnout. (ALMEIDA et al., 2016; BARBOSA et al., 2018; BATISTA et al., 2010; PACHECO et al., 2017). Esse último, por sua vez, esteve fortemente relacionado com a ausência em atividades ocupacionais e diminuição da produtividade acadêmica, o que resultou em aumento da exaustão emocional e piora do bem estar biopsicossocial entre professores e alunos acometidos pela síndrome (ALMEIDA et al., 2016; ALVES; OLIVEIRA; PARO, 2019; CALCIDES et al., 2019). Observou-se uma forte correlação do Burnout com as seguintes variáveis: "não enxergar o curso como fonte de prazer" (COSTA et al., 2012) "faltar atividades avaliativas"; "considerar abandonar a graduação" (ALMEIDA et al., 2016); falta de otimismo; "não estar realizado como estudante" (BONI et al., 2018) pior desempenho acadêmico (CALCIDES et al., 2019); "achar a profissão menos interessante do que quando começou a trabalhar"; e "desejo de mudar de profissão" (BATISTA et al., 2010). Todas as variáveis mencionadas estão diretamente associadas com os níveis de realização acadêmica e pessoal. Esses últimos, por sua vez, foram identificados como preditores positivos da qualidade de vida de docentes e discentes do Brasil. (ALMEIDA et al., 2016; ALVES; OLIVEIRA; PARO, 2019; BATISTA et al., 2010; PAGNIN \& QUEIROZ, 2015).

A sensação de eficácia acadêmica/ realização profissional é associada com melhora do bem-estar psicológico, saúde física e relações sociais de indivíduos acometidos pelo Burnout (ALVES; OLIVEIRA; PARO, 2019; PAGNIN \& QUEIROZ, 2015; PARO et al., 2014). Professores e alunos insatisfeitos com suas atividades ocupacionais estão susceptíveis a comprometimento do sono, alterações do humor, ansiedade e depressão, o que contribui para piora da qualidade de vida nestes sujeitos (ALVES; OLIVEIRA; PARO, 2019; PARO et al., 2014).A literatura nacional indica que o bem estar acadêmico não é determinado apenas pelo caráter intrínseco de atividades ocupacionais, sendo também dependente da forma com que a instituição educacional lida com professores e alunos. Desse modo, a preocupação em fornecer condições adequadas de trabalho e um ambiente adequado de aprendizado influencia diretamente nos níveis de Burnout e qualidade de vida da comunidade acadêmica (ALVES; OLIVEIRA; PARO, 2019; BATISTA et al., 2010; PAGNIN \& QUEIROZ, 2015). Ressalta-se que, muitas vezes, os professores assumem funções contra sua vontade para atender a solicitações da instituição em que trabalha. Assim, esses indivíduos experimentam o conflito de papéis no exercício de sua profissão, o que foi fortemente correlacionado com os níveis de Burnout (CARLOTTO \& CÂMARA, 2017). A referida síndrome também foi relacionada à falta de participação de professores em decisões institucionais (CARLOTTO \& PALAZZO, 2006) e à insatisfação de alunos com as estratégias de ensino da sua faculdade (CALCIDES et al., 2019). Esses achados demonstram uma forte associação de questões institucionais com o Burnout e qualidade de vida do corpo acadêmico. Tabeleão, Tomasi e Neves (2011) identificaram que quando comparados com colegas da rede municipal, os professores da rede estadual de ensino apresentam maiores índices de exaustão e despersonalização, o que determinou um maior comprometimento da qualidade de vida destes profissionais. Esses achados ressaltam a forte associação entre Burnout e bem estar biopsicossocial de 
professores. Ademais, é possível que as diferenças observadas entre profissionais da rede estadual e municipal foram provenientes da forma com que a instituição educacional lida com o seu corpo docente. O período da graduação é um dos fatores ocupacionais mais relacionados à qualidade de vida de estudantes universitários. Ao comparar as pontuações obtidas no questionário WHOQOL-BREF para alunos de medicina, Ramos-Dias et al. (2010) constataram que o primeiro ano do curso é um preditor negativo de qualidade de vida no domínio de relações sociais. Os escores obtidos por alunos do primeiro e sexto ano foram, respectivamente, 70,2 e 77,8 (RAMOSDIAS et al., 2010). Ao avaliar alunos de uma escola médica que utilizava metodologias ativas de ensino, Boni et al. (2018) identificaram níveis aumentados de Burnout nos estudantes do primeiro ano, o que pode explicar a menor qualidade de vida neste período da graduação. Os autores sugeriram que alunos matriculados em instituições com metodologias de aprendizado diferentes das experimentadas no ensino fundamental tem maior risco de desenvolver transtornos da saúde mental (BONI et al., 2018). Contrapondo os dados supracitados, outros estudos sugeriram que o pico de incidência do Burnout acontece no último ano da graduação. Acredita-se que a transição dos ciclos mais básicos para o internato médico associa-se com sentimentos de ansiedade, incerteza e medo, o que pode contribuir para susceptibilidade ao Burnout no final da graduação em medicina. Desse modo, é possível que a saúde mental dos acadêmicos se deteriore à medida que o curso progride, com o ápice do desgaste mental ocorrendo nos estágios finais da formação (ALMEIDA et al., 2016; COSTA et al., 2012). Ressalta-se que, situações vivenciadas ao longo da graduação podem ser gatilhos críticos para o desenvolvimento da síndrome e comprometimento da qualidade de vida de discentes. $\mathrm{O}$ contato com pacientes em estado críticos de saúde é um possível gatilho para doença entre os alunos de medicina. (ALMEIDA et al., 2016).

Fatores psicológicos: A análise dos dados obtidos pela literatura revelam que o comprometimento do bem-estar psicológico de professores e alunos resulta da exposição aos mesmos estressores ocupacionais associados com o desenvolvimento do Burnout (ALMEIDA et al., 2016 ; ALVES; OLIVEIRA; PARO, 2019; CARLOTTO \& PALAZZO, 2006; COSTA et al., 2012; GILMONTE; CARLOTTO; CÂMARA, 2011; PACHECO et al., 2017).

Estudos nacionais identificaram que, além de correlacionarem-se com maior incidência de Burnout, fatores como falta de motivação, sobrecarga acadêmica e ausência de apoio emocional contribuem para aumento da incidência de doenças que comprometem a saúde mental de professores e alunos. Desse modo, transtornos como ansiedade, estresse e depressão exercem um papel crucial na perda do bem estar biopsicossocial na comunidade acadêmica (ALMEIDA et al., 2016 ; ALVES; OLIVEIRA; PARO, 2019; ; CARLOTTO \& PALAZZO, 2006; COSTA et al., 2012; PACHECO et al., 2017). Demonstrou-se alta prevalência de transtornos da saúde mental em alunos de medicina. Os percentuais encontrados foram de 30,6\% para depressão; 13,1\% para Burnout; 49,9\% de estresse; e 32,9\% de ansiedade. (PACHECO et al., 2017). O fato de ter escolhido uma profissão que lida diretamente com vidas e tem pouca tolerância a erros é proposto para explicar a suscetibilidade ao adoecimento mental entre futuros médicos (ALMEIDA et al., 2016; BONI et al., 2018). Quando comparados com pares da mesma idade na população geral, os acadêmicos de medicina apresentam maior prevalência de Burnout e depressão (BARBOSA et al., 2018; COSTA et al., 2012; PACHECO et al., 2017). Por sua vez, a presença de sintomas depressivos foi fortemente relacionada ao agravamento dos domínios psicológico, social e ambiental do bem-estar destes estudantes (PAGNIN \& QUEIROZ, 2015). Os escores de sofrimento pessoal também foram preditores negativos da qualidade vida no ambiente universitário (PARO et al., 2014). Outros fatores relacionados com comprometimento do bem-estar psicológico no meio acadêmico são: relações pessoais negativas no ambiente de trabalho (BATISTA et al., 2010); incertezas sobre o futuro profissional; (ALMEIDA et al., 2016; BARBOSA et al., 2018; PACHECO et al., 2017) e insegurança em habilidades clínicas (COSTA et al., 2012). Embora não tenham sido identificadas diretamente como preditores de qualidade de vida, todas essas variáveis aumentam o risco de Burnout, o que pode afetar negativamente o bem-estar biopsicossocial no ambiente universitário. As possíveis repercussões da síndrome de Burnout no bem-estar biopsicossocial de professores e alunos incluem comprometimento do sono, uso de drogas ilícitas, problemas conjugais, estresse, ansiedade, depressão e suicídio (ALMEIDA et al., 2016 ; ALVES, OLIVEIRA, PARO, 2019; COSTA et al., 2012; PACHECO et al., 2017). A exaustão emocional e a despersonalização são dimensões da doença fortemente relacionadas com o comprometimento psicológico no meio acadêmico (ALVES; OLIVEIRA; PARO, 2019; PAGNIN \& QUEIROZ, 2015). A preocupação empática é tanto um preditor positivo de qualidade de vida (PARO et al., 2014) quanto um fator de risco para síndrome de Burnout (LOPES; NIHEI, 2020) entre estudantes universitários. A empatia é um valor essencial para o profissionalismo médico, sendo definida como a capacidade de compreender, entender e responder as experiências e emoções de outras pessoas. (PARO et al., 2014). A empatia também foi inversamente relacionada com o risco de desenvolver a síndrome de Burnout (PARO et al., 2014), a qual, por sua vez, esteve associada com comprometimento da qualidade do atendimento médico (BARBOSA et al., 2018; PACHECO et al., 2017).

Fatores fisiológicos: saúde física e relações sociais: Alguns estudos nacionais demonstraram correlações significativas entre comorbidades, Burnout e qualidade de vida no meio acadêmico. Araújo et al. (2020) identificaram risco aumentado para síndrome entre professores acometidos por doenças crônicas (ARAÚJO et al., 2020). Em contrapartida, Batista et al. (2010) relacionaram a história de afastamento por motivo de saúde com o Burnout nestes profissionais. (BATISTA et al., 2010). Costa \& Silva (2012) identificaram que a maior parte $(58 \%)$ dos professores que atuam no estado do Paraná tem ou tiveram problemas de saúde como resultado de sua profissão. Dentre os problemas mais frequentes estiveram Burnout, cefaleia e problemas vocais, sendo que, o comportamento negativo dos alunos e a falta de respeito contribuíram diretamente para adoecimento ocupacional (COSTA \& SILVA, 2012). Solis\& Lotufo-Neto (2019) constataram que a presença de doenças crônicas é um preditor negativo da qualidade de vida entre acadêmicos de medicina (SOLIS \& LOTUFO-NETO, 2019). Por sua vez, Boni et al. (2018) demonstraram que a autopercepção de saúde está positivamente associada com os níveis de Burnout nestes estudantes. A resposta anômala ao estresse emocional crônico e a exaustão emocional que ocorrem na síndrome de Burnout explicam o comprometimento da saúde física entre professores e alunos acometidos pela doença (ALVES; OLIVEIRA; PARO, 2019; BARBOSA et al., 2018; Costa \& Silva, 2012; PAGNIN \& QUEIROZ, 2015).

Ao analisar membros de um corpo docente, Alves, Oliveira e Paro (2019) identificaram a necessidade de mais tempo para relaxar após o trabalho como um preditor negativo da qualidade de vida destes profissionais. (ALVES; OLIVEIRA; PARO, 2019) Mota et al. (2019) encontraram correlação positiva entre exaustão psíquica e distúrbios da voz em docentes. (MOTA et al., 2019). Os portadores da síndrome que também são acometidos por doenças crônicas podem experimentar uma diminuição significativa de sua capacidade funcional, o que pode afetar o desempenho em atividades ocupacionais e comprometer ainda mais a qualidade de vida destes sujeitos (ALVES; OLIVEIRA; PARO, 2019; BARBOSA et al., 2018; GIANNINI; LATORRE; FERREIRA, 2013; MOTA et al., 2019). Giannini, Latorre e Ferreira (2013) observaram associação entre estresse ocupacional, distúrbios da voz e perda da capacidade para o trabalho. (GIANNINI; LATORRE; FERREIRA, 2013). Além de comprometer a saúde mental de professores e alunos, a indução do estresse crônico é associada com a adoção de comportamentos de risco, como tabagismo, alcoolismo, sedentarismo e hábitos alimentares inadequados, que podem resultar em aumento da incidência de distúrbios cardiometabólicos como hipertensão, obesidade e diabetes. (BARBOSA et al., 2018; Costa \& Silva, 2012; PAGNIN \& QUEIROZ, 2015). O comprometimento do sono foi outro fator identificado como um preditor negativo da qualidade de vida no meio acadêmico (PAGNIN \& QUEIROZ, 2015; SOLIS \& 
LOTUFO-NETO, 2019). Segundo Pagnin\& Queiroz (2015), as repercussões do Burnout e a baixa qualidade de sono explicam $22 \%$ da diminuição no bem-estar físico e $21 \%$ da redução no bem-estar psicológico entre estudantes de medicina no Brasil. (PAGNIN \& QUEIROZ, 2015). A privação de sono é fortemente associada com susceptibilidade para síndrome de Burnout na literatura nacional (ALMEIDA et al., 2016; ALVES; OLIVEIRA; PARO, 2019; BARBOSA et al., 2018; NEVES \& RIBEIRO, 2016; PACHECO et al., 2017).

As atividades realizadas fora do ambiente acadêmico também influenciam no desenvolvimento da síndrome de Burnout. Desse modo, fatores como baixa frequência de encontros familiares, uso de drogas lícitas, tempo de lazer e influência da profissão na vida pessoal aumentam a suscetibilidade para síndrome e podem impactar negativamente na qualidade de vida de docentes e discentes (BONI et al., 2018; BATISTA et al., 2010; CALCIDES et al., 2019). Ao comparar o bem estar biopsicossocial entre estudantes de medicina do primeiro e último ano, Ramos-Dias et al. (2010) observaram que a única diferença estatisticamente relevante no questionário WHOQOL-BREF ocorreu no domínio das relações sociais, onde foram identificadas menores escores para os alunos do primeiro ano $(70,2$ versus 77,8$)$. Os autores sugeriram que a adaptação à nova vida acadêmica afetou negativamente as relações sociais destes acadêmicos. (RAMOS-DIAS et al., 2010). Conclui-se, portanto, que diversos fatores sociodemográficos, laborais e psicossociais associados com o desenvolvimento do Burnout entre docentes e discentes do Brasil também estão correlacionados com uma menor percepção de qualidade de vida nestes sujeitos. Assim, é possível que a referida síndrome explique, pelo menos em parte, a associação dessas variáveis com o comprometimento do bem-estar biopsicossocial de professores e alunos. Assim sendo, considerando que múltiplos e complexos fatores estejam correlacionados com o desenvolvimento do Burnout entre professores e alunos, percebe-se que é de extrema importância que as instituições de ensino superior desenvolvam estratégias para identificação e gestão de fatores estressores que impactam negativamente na saúde mental de discentes e docentes, contribuindo para a alta prevalência do Burnout em diversas instituições de ensino do Brasil. Ressalta-se que, ao atuar sobre os diversos agentes estressores presentes no ambiente acadêmico é possível melhorar significativamente as relações de ensino-aprendizagem e obter melhores resultados pedagógicos, o que contribui para prevenção do Burnout e melhora da percepção de qualidade de vida entre professores e alunos.

\section{CONSIDERAÇÕES FINAIS}

A Síndrome de Burnout possui prevalência significativa entre estudantes e professores nos diversos níveis educacionais. As diversas dimensões afetadas no esgotamento profissional e estudantil são preditores negativos de qualidade de vida no meio acadêmico. $\mathrm{O}$ maior número de estudos brasileiros abordando o Burnout em estudantes do curso médico podem indicar maior exposição de tais estudantes à fatores estressores. $\mathrm{O}$ acometimento de docentes também foi constatado, tanto em níveis médio e básico de ensino, como em ensino superior. A ocorrência da Síndrome de Burnout possui relação direta com menor rendimento estudantil e profissional, além gerar sentimentos de insuficiência, desejo de abandono do trabalho e/ou estudo, além de quadros depressivos e transtornos de ansiedade.

\section{REFERÊNCIAS}

Almeida, G. D. C., Souza, H. R. D., Almeida, P. C. D., Almeida, B. D. C. et al. A prevalência da síndrome de burnout em estudantes de medicina. Archives of Clinical Psychiatry (São Paulo), 43, n. 1, p. 6-10, 2016-02 2016.

Alves, P. C., Oliveira, A. F., Paro, H. Quality of life and burnout among faculty members: How much does the field of knowledge matter? PLoS One, 14, n. 3, p. e0214217, 2019.

Barbosa, M. L., Ferreira, B. L. R., Vargas, T. N., Ney DA Silva, G. M. et al. Burnout Prevalence and Associated Factors Among
Brazilian Medical Students. Clin Pract Epidemiol Ment Health, 14, p. 188-195, 2018.

Batista, J. B., Carlotto, M. S., Coutinho, A. S., AUGUSTO, L. G. [Prevalence of Burnout Syndrome and sociodemographic and work factors of elementary education teachers of the City of João Pessoa]. Rev Bras Epidemiol, 13, n. 3, p. 502-512, Sep 2010.

Boni, R., Paiva, C. E., De Oliveira, M. A., Lucchetti, G. et al. Burnout among medical students during the first years of undergraduate school: Prevalence and associated factors. PLoS One, 13, n. 3, p. e0191746, 2018.

Brasil. Ministério da Fazenda. $1{ }^{\circ}$ Boletim Quadrimestral sobre benefícios por Incapacidade. Adoecimento Mental e Trabalho: A concessão de benefícios por incapacidade relacionada a transtornos mentais e comportamentais entre 2012 e 2016. Brasília: Ministério da Fazenda; 2017.

Calcides, D. A. P., Didou, R. D. N., Melo, E. V., Oliva-Costa, E. F. Burnout Syndrome in medical internship students and its prevention with Balint Group. Rev Assoc Med Bras (1992), 65, n. 11, p. 1362-1367, Nov 2019.

Campos, J. A., Maroco, J. [Maslach Burnout Inventory - Student Survey: Portugal-Brazil cross-cultural adaptation]. Rev Saude Publica, 46, n. 5, p. 816-824, Oct 2012.

Carlotto, M. S., Câmara, S. G. Burnout Syndrome Profiles among Teachers. Escritos de Psicología (Internet), 10, n. 3, p. 159-166, 2017-12 2017.

Carlotto, M. S., Palazzo Ldos, S. [Factors associated with burnout's syndrome: an epidemiological study of teachers]. Cad Saude Publica, 22, n. 5, p. 1017-1026, May 2006.

Costa, B. E., Silva, N. L. Analysis of environmental factors affecting the quality of teacher's life of public schools from Umuarama. Work, 41 Suppl 1, p. 3693-3700, 2012.

Costa, E. F. D. O., Santos, S. A., Santos, A. T. R. D. A., Melo, E. V. D. et al. Burnout Syndrome and associated factors among medical students: a cross-sectional study. Clinics, 67, n. 6, p. 573-580, 2012/00 2012.

De Araújo Leite, T. I., Costa Fernandes, J. P., DA Costa Araújo, F. L., De Brito Fernandes Pereira, X. et al. Prevalence and factors associated with burnout among university professors. Rev Bras Med Trab, 17, n. 2, p. 170-179, 2019.

De Brito Mota, A. F., Giannini, S. P. P., De Oliveira, I. B., Paparelli, R. et al. Voice Disorder and Burnout Syndrome in Teachers. J Voice, 33, n. 4, p. 581.e587-581.e516, Jul 2019.

Giannini, S. P., Latorre MDO, R., Ferreira, L. P. Factors associated with voice disorders among teachers: a case-control study. Codas, 25, n. 6, p. 566-576, 2013.

Gil-Monte, P. R., Carlotto, M. S., Gonçalves Câmara, S. Prevalence of burnout in a sample of Brazilian teachers. The European Journal of Psychiatry, 25, n. 4, p. 205-212, 2011-12 2011.

Lopes, A. R., Nihei, O. K. Burnout among nursing students: predictors and association with empathy and self-efficacy. Revista Brasileira de Enfermagem, 73, n. 1, 2020-02-10 2020.

Mendes, K. D. S; Silveira, R. C. C; Galvao, C. M. Uso de gerenciador de referências bibliográficas na seleção dos estudos primários em revisão integrativa. Texto contexto - enferm., Florianópolis, v. 28, n. e20170204, 2019.

Neves, C. P. D., Ribeiro, D. M. Burnout em estudantes de graduação em Odontologia. Rev. ABENO, 16, n. 1, p. 39-49, 2016/00 2016.

Pacheco, J. P., Giacomin, H. T., TAM, W. W., Ribeiro, T. B. et al. Mental health problems among medical students in Brazil: a systematic review and meta-analysis. Braz J Psychiatry, 39, n. 4, p. 369-378, Oct-Dec 2017.

Pagnin, D., De Queiroz, V. Influence of burnout and sleep difficulties on the quality of life among medical students. Springerplus, 4, p. 676, 2015.

Paro, H. B., Silveira, P. S., Perotta, B., Gannam, S. et al. Empathy among medical students: is there a relation with quality of life and burnout? PLoS One, 9, n. 4, p. e94133, 2014.

Ramos-Dias, J. C., Libardi, M. C., Zillo, C. M., Igarashi, M. H. et al. Qualidade de vida em cem alunos do curso de Medicina de Sorocaba - PUC/SP. Revista Brasileira de Educação Médica, 34, n. 1, p. 116-123, 2010-03 2010. 
Reis, E. J. F. B. D., Araújo, T. M. D., Carvalho, F. M., Barbalho, L. et al. Docência e exaustão emocional. Educação \& Sociedade, 27, n. 94, p. 229-253, 2006-04 2006.

Solis, A. C., Lotufo-Neto, F. Predictors of quality of life in Brazilian medical students: a systematic review and meta-analysis. Braz J Psychiatry, 41, n. 6, p. 556-567, Nov-Dec 2019.
Tabeleão, V. P., Tomasi, E., Neves, S. F. Qualidade de vida e esgotamento profissional entre docentes da rede pública de Ensino Médio e Fundamental no Sul do Brasil. Cadernos de Saúde Pública, 27, n. 12, p. 2401-2408, 2011-12 2011.

Tomaschewski-Barlem, J. G., Lunardi, V. L., Lunardi, G. L., Barlem, E. L. et al. Burnout syndrome among undergraduate nursing students at a public university. Rev Lat Am Enfermagem, 22, n. 6, p. 934-941, Nov-Dec 2014. 